PROCEEDINGS OF THE

AMERICAN MATHEMATICAL SOCIETY

Volume 125, Number 5, May 1997, Pages 1503-1509

S 0002-9939(97)03692-7

\title{
GENERAL HELICES AND A THEOREM OF LANCRET
}

\author{
MANUEL BARROS \\ (Communicated by Christopher Croke)
}

\begin{abstract}
We present a theorem of Lancret for general helices in a 3-dimensional real-space-form which gives a relevant difference between hyperbolic and spherical geometries. Then we study two classical problems for general helices in the 3-sphere: the problem of solving natural equations and the closed curve problem.
\end{abstract}

\section{INTRODUCTION}

A curve of constant slope or general helix in Euclidean space $R^{3}$ is defined by the property that the tangent makes a constant angle with a fixed straight line (the axis of the general helix). A classical result stated by M. A. Lancret in 1802 and first proved by B. de Saint Venant in 1845 (see [St] for details) is: A necessary and sufficient condition that a curve be a general helix is that the ratio of curvature to torsion be constant.

Given two functions of one parameter (potentially curvature and torsion parametrized by arclength) one might like to find an arclength parametrized curve for which the two functions work as the curvature and the torsion. This problem, known as solving natural equations, is generally achieved by solving a Riccati equation, [St]. However, the natural equations for a general helix can be integrated by quadrature using the fact that such a curve is a geodesic on a general cylinder (see [St]).

The curvature and the torsion of an arclength parametrized closed curve are periodic functions of the arclength. However this necessary condition is not a sufficient condition, as general helices show. N. V. Efimow [Ef] and W. Fenchel [Fe] stated the so called closed curve problem: Find (explicit) necessary and sufficient conditions that determine when, given two periodic functions with the same period, the integral curve is closed. This is an open problem in elementary differential geometry.

In this short note, we use the concept of Killing vector field along a curve $\gamma$ in a 3-dimensional real-space-form $M$ (this concept was introduced by J. Langer and D. A. Singer when studying elastic curves in real-space-forms, [LS1], [LS2]) to define the concept of general helix in $M$. Of course this is the natural extension of that for general helices in Euclidean space.

Received by the editors July 26, 1995 and, in revised form, November 14, 1995.

1991 Mathematics Subject Classification. Primary 53C40, 53A05.

Key words and phrases. General helix, theorem of Lancret, Hopf cylinder.

Partially supported by DGICYT Grant No. PB94-0750.

(C) 1997 American Mathematical Society 
Then we prove the Lancret Theorem for general helices in a space-form, $M$. While the original Lancret Theorem is very elementary, the extension to spaceforms is a bit more subtle than one might suppose, as evidenced by the difference between the spherical and hyperbolic cases. The spherical case (Theorem 3) is nicely analogous to the Euclidean case (Theorem 2). On the other hand, there are no nontrivial general helices in the hyperbolic case (Theorem 1).

The more explicit realization of general helices in the sphere (Theorems 4, 5) makes essential use of the Hopf map $\Pi: S^{3} \longrightarrow S^{2}$. It is used to define on $S^{3}$ a class of flat surfaces. Indeed, just consider $M_{\beta}=\Pi^{-1}(\beta)$, the Hopf cylinder over an immersed curve $\beta$ in $S^{2}$, [Pi]. Then we give a geometric approach to the problem of solving natural-equations for general helices in 3 -sphere. We prove that a fully immersed curve in $S^{3}$ is a general helix if and only if it is a geodesic in some Hopf cylinder in $S^{3}$.

However, general helices in $S^{3}$ behave quite differently than those in Euclidean space. In fact, when we study the closed-curve problem for general helices in $S^{3}$, we prove: There exists a rational one-parameter family of closed general helices living in the Hopf torus shaped on any immersed closed curve in the 2-sphere (see Corollary 1).

Finally we make some speculations about the interest of general helices. These curves certainly arise in a broader context: the interplay between geometry and integrable Hamiltonian systems. Embedded in the LIH (localized induction hierarchy) is a natural geometric evolution on general helices inducing an mKdV (modified Korteweg-de Vries) curvature evolution equation. The role of general helices here is probably similar to that of curves of constant torsion or constant natural curvature (see [LP] for details).

The author would like to express his thanks to the referee for valuable comments and suggestions.

\section{General helices}

Let $\gamma=\gamma(t): I \subset R \longrightarrow M$ be an immersed curve in a 3-dimensional realspace-form $M$ with sectional curvature $c$. The unit tangent vector field of $\gamma$ will be denoted by $T$ and the speed of $\gamma$ will be $v(t)=\left|\gamma^{\prime}(t)\right|$. Also $\kappa>0$ and $\tau$ will denote the curvature and the torsion of $\gamma$, respectively. Therefore if $\{T, N, B\}$ is the Frenet frame of $\gamma$ and $\bar{\nabla}$ is the Levi-Civita connection of $M$, then one can write the Frenet equations of $\gamma$ as

$$
\begin{aligned}
\bar{\nabla}_{T} T & =\kappa N, \\
\bar{\nabla}_{T} N & =-\kappa T+\tau B, \\
\bar{\nabla}_{T} B & =-\tau N .
\end{aligned}
$$

We consider variations $\Gamma=\Gamma(t, z): I \times(-\varepsilon, \varepsilon) \longrightarrow M$ with $\Gamma(t, 0)=\gamma(t)$. Associated with $\Gamma$ is the variational field $V(t)=\frac{\partial \Gamma}{\partial z}(t, 0)$ along $\gamma$. We will use the notation $V=V(t, z), T=T(t, z), v=v(t, z)$, etc. with the obvious meanings. Finally we use $s$ to denote the arclength parameter of the $t$-curves in variation $\Gamma$ and write $v(s, z), \kappa^{2}(s, z), \tau^{2}(s, z)$, etc. for the corresponding reparametrizations. 
According to [LS1], a vector field $V(s)$ along $\gamma(s)$ is a Killing vector field along $\gamma$ if

$$
\left.\frac{\partial v}{\partial z}\right|_{z=0}=\left.\frac{\partial \kappa^{2}}{\partial z}\right|_{z=0}=\left.\frac{\partial \tau^{2}}{\partial z}\right|_{z=0}=0 .
$$

This is well-defined in the sense that it does not depend on the $V$-variation of $\gamma$ one chooses to compute the derivatives involved in (1). Indeed, just use standard arguments (see [LS1]) to get

$$
\begin{aligned}
\left.\frac{\partial v}{\partial z}\right|_{z=0}= & \left\langle\bar{\nabla}_{T} V, T\right\rangle v=0, \\
\left.\frac{\partial \kappa^{2}}{\partial z}\right|_{z=0}= & 2 \kappa\left\langle\bar{\nabla}_{T}^{2} V, N\right\rangle-4 \kappa^{2}\left\langle\bar{\nabla}_{T} V, T\right\rangle+2 c \kappa\langle V, N\rangle=0, \\
\left.\frac{\partial \tau^{2}}{\partial z}\right|_{z=0}= & \frac{2 \tau}{\kappa}\left\langle\bar{\nabla}_{T}^{3} V, B\right\rangle-\frac{2 \kappa^{\prime} \tau}{\kappa^{2}}\left\langle\bar{\nabla}_{T}^{2} V+c V, B\right\rangle \\
& +\frac{2 \tau\left(c+\kappa^{2}\right)}{\kappa}\left\langle\bar{\nabla}_{T} V, B\right\rangle-2 \tau^{2}\left\langle\bar{\nabla}_{T} V, T\right\rangle=0,
\end{aligned}
$$

where $\langle$,$\rangle denotes the Riemannian metric of M$ and $\kappa^{\prime}=\frac{\partial \kappa}{\partial s}(s, 0)$.

These equations constitute a linear system in $V$ whose solution space is 6dimensional. Obviously the restriction to $\gamma$ of any Killing field of $M$ gives a Killing vector field along $\gamma$. In particular, if $M$ is simply connected, then the dimension of its isometry group is 6 , and then we have the following lemma due to J. Langer and D. A. Singer (see also [LS2]).

Lemma 1. Let $M$ be a complete, simply connected real-space-form and $\gamma$ an immersed curve in $M$. A vector field $V$ on $\gamma$ is a Killing vector field along $\gamma$ if and only if it extends to a Killing field $\tilde{V}$ on $M$.

From now on $M$ will be complete and simply connected. Also, without loss of generality we will assume $c=-1,0,+1$.

A curve $\gamma(s)$ in $M$ will be called a general helix if there exists a Killing vector field $V(s)$ with constant length along $\gamma$ and such that the angle between $V$ and $\gamma^{\prime}$ is a non-zero constant along $\gamma$. We will say that $V$ is an axis of the general helix $\gamma$. As we will see, this definition is the natural extension of that for general helices in $R^{3}$, in which an axis works as a straight line (see for instance [MP]).

Obvious examples of general helices are:

(1) Any curve in $M$ with $\tau \equiv 0$. In this case just take $V=B$ as an axis.

(2) The curves with both $\kappa$ and $\tau$ being nonzero constants (we call them helices). In this case $V(s)=\cos \theta T(s)+\sin \theta B(s)$ with $\cot \theta=\frac{\tau^{2}-c}{\tau \kappa}$ works as an axis.

\section{The THEOREM OF LANCRET}

Let $\gamma(s)$ be a general helix with axis $V(s)$; without loss of generality we may assume that $|V(s)|=1$. Then

$$
V(s)=\cos \theta T(s)+\sin \theta B(s),
$$

where $\theta$ denotes the angle between $V(s)$ and $T(s)=\gamma^{\prime}(s)$. 
We use the last formula and the Frenet equations of $\gamma$ to obtain

(6) $\bar{\nabla}_{T} V=(\kappa \cos \theta-\tau \sin \theta) N$,

(7) $\bar{\nabla}_{T}^{2} V=\left(\kappa^{\prime} \cos \theta-\tau^{\prime} \sin \theta\right) N-\kappa(\kappa \cos \theta-\tau \sin \theta) T+\tau(\kappa \cos \theta-\tau \sin \theta) B$.

Next we put (6) and (7) in (3), getting

$$
\kappa\left(\kappa^{\prime} \cos \theta-\tau^{\prime} \sin \theta\right)=0 .
$$

Since $\kappa$ is assumed to be positive, (8) implies that $\kappa \cos \theta-\tau \sin \theta$ must be a constant, and consequently we have

$$
\tau=b \kappa+a,
$$

where $b=\cot \theta$

To determine the constant $a$, we use the fact that $\bar{\nabla}_{T}^{2} V$ has no $N$-component to compute

$$
\bar{\nabla}_{T}^{3} V=a \kappa^{\prime} \sin \theta T-a \tau^{\prime} \sin \theta B+a\left(\kappa^{2}+\tau^{2}\right) \sin \theta N .
$$

Finally we put all this information in (4) to get

$$
\tau \sin \theta\left(a\left(\kappa \tau^{\prime}-\tau \kappa^{\prime}\right)+c \kappa^{\prime}\right)=0 .
$$

Because of the examples, we can consider $\tau \neq 0$, and so we use (9) and (11) to obtain

$$
\kappa^{\prime}\left(a^{2}-c\right)=0 .
$$

As a consequence, $\kappa$ is constant (which is included in (9)) or $a^{2}=c$. According to the values of $c$, we have the following cases:

Theorem 1 (The Lancret theorem in the hyperbolic space). A curve $\gamma$ in $H^{3}(-1)$ is a general helix if and only if either

(1) $\tau \equiv 0$ and $\gamma$ is a curve in some hyperbolic plane $H^{2}(-1)$, or

(2) $\gamma$ is a helix in $H^{3}(-1)$.

In contrast we have the classical theorem of Lancret in Euclidean space and its spherical version.

Theorem 2 (The Lancret theorem in Euclidean space). A curve in $R^{3}$ is a general helix if and only if there exists a constant $b$ such that $\tau=b \kappa$.

Theorem 3 (The Lancret theorem in the sphere). A curve $\gamma$ in $S^{3}(1)$ is a general helix if and only if either

(1) $\tau \equiv 0$ and $\gamma$ is a curve in some unit 2-sphere $S^{2}(1)$, or

(2) there exists a constant b such that $\tau=b \kappa \pm 1$.

We only need to prove the if parts of Theorems 2 and 3 . If $\kappa$ and $\tau$ are constrained according to any of the above mentioned statements, we define $\theta$ to satisfy $\cot \theta=b$ and then consider $V(s)=\cos \theta T(s)+\sin \theta B(s)$. It is clear that $V$ has constant length and $\theta$ is the angle between $V$ and $T=\gamma^{\prime}$ along $\gamma$. Now it is not difficult to compute $\bar{\nabla}_{T} V, \bar{\nabla}_{T}^{2} V$ and $\bar{\nabla}_{T}^{3} V$ and then to see that $V$ satisfies (2), (3) and (4). This proves that $V(s)$ is a Killing vector field along $\gamma$. 


\section{General helices And Hopf Cylinders in the 3-Sphere}

It is well-known that general helices in Euclidean space can be regarded as geodesics in right cylinders (of course the converse also hold). In this section we show a similar result for general helices lying fully in the 3 -sphere.

We start by recalling a nice description (due to U. Pinkall, [Pi]) of the Hopf map $\Pi: S^{3}(1) \longrightarrow S^{2}\left(\frac{1}{2}\right)$ (here $S^{n}(r)$ denotes the $n$-sphere of radius $r>0$ and $S^{2}\left(\frac{1}{2}\right)$ is identified with the one dimensional complex projective space $C P^{1}$ with constant holomorphic sectional curvature 4$)$. We identify $S^{3}(1)$ with the set of unit quaternions $\{q \in H, q \bar{q}=1\}$ and then $S^{2}\left(\frac{1}{2}\right)$ with the 2-sphere of radius $\frac{1}{2}$ in the subspace of $H$ spanned by $1, j$ and $k$. Now we denote by $q \mapsto \tilde{q}$ the antiautomorphism of $H$ that fixes $1, j$ and $k$ but sends $i$ to $-i$. Then $\Pi(q)=\frac{1}{2} \tilde{q} q$, (see $[\mathrm{Pi}]$ for properties of $\Pi$ ). In particular $\Pi$ is a Riemannian submersion, and so we will follow the notation and terminology of [O]. Therefore, given $p \in S^{3}(1)$, the tangent space $T_{p} S^{3}(1)$ splits into the horizontal plane (which is isometric, via $d \Pi_{p}$, to $\left.T_{\Pi(p)} S^{2}\left(\frac{1}{2}\right)\right)$ and the vertical line (which is the tangent line to the fiber through $p)$.

Let $\bar{\nabla}$ and $\nabla$ be the Levi-Civita connections of $S^{3}(1)$ and $S^{2}\left(\frac{1}{2}\right)$, respectively, and denote by overbars the horizontal lifts of corresponding objects on the base. Then we have

$$
\begin{aligned}
& \bar{\nabla}_{\bar{X}} \bar{Y}=\nabla_{X}^{-} Y-(\langle J X, Y\rangle \circ \Pi) V, \\
& \bar{\nabla}_{\bar{X}} V=\bar{\nabla}_{V} \bar{X}=i \bar{X}, \\
& \bar{\nabla}_{V} V=0
\end{aligned}
$$

where $V$ is a unit vertical vector field and $J$ denotes the standard complex structure on $S^{2}\left(\frac{1}{2}\right)$. Notice that (15) expresses the geodesic nature of the fibers.

Let $\beta: I \subset R \longrightarrow S^{2}\left(\frac{1}{2}\right)$ be a unit speed curve with Frenet frame $\left\{\beta^{\prime}, \xi\right\}$ and curvature function $\lambda$. Consider a horizontal lift $\bar{\beta}$ of $\beta$ with Frenet frame $\left\{\bar{\beta}^{\prime}, \xi^{*}, \eta^{*}\right\}$ and curvatures $\lambda^{*}$ and $\mu^{*}$. Then we combine $(13),(14),(15)$ with the Frenet equations of both $\beta$ and $\bar{\beta}$ to prove that $\lambda^{*}=\lambda \circ \Pi=\bar{\lambda}$ and $\xi^{*}=\bar{\xi}$. In particular, $\xi^{*}$ lies in the horizontal distribution along $\bar{\beta}$. Also it is not difficult to see that $\mu^{*}= \pm 1$ and $\eta^{*}= \pm V$; that is, the binormal $\eta^{*}$ of $\bar{\beta}$ and the unit tangent to the fibers through each point of $\bar{\beta}$ agree.

By pulling back via $\Pi$ the curve $\beta$, we obtain its total lift $M_{\beta} \subset S^{3}(1)$, which is proved to be a flat surface in $S^{3}(1)$, [Pi]. We call $M_{\beta}$ the Hopf cylinder over $\beta$. It can be parametrized as follows $X: I \times R \longrightarrow S^{3}(1)$ defined by

$$
X(t, z)=e^{i z} \bar{\beta}(t)=\cos z \bar{\beta}(t)+\sin z V(t)
$$

Notice that in this parametrization, the t-curves are the horizontal lifts of $\beta$ while the $z$-curves correspond to the fibers. Both families of curves are arclength parametrized and mutually orthogonal; futhermore, they are geodesics in $M_{\beta}$. U. Pinkall also showed that the Hopf cylinder $M_{\beta}$ associated with a closed curve $\beta$ of length $L$ enclosing an oriented area $A$ in $S^{2}\left(\frac{1}{2}\right)$ is isometric to the flat torus $R^{2} / \Lambda$, where $\Lambda$ is the lattice generated by $(2 A, L)$ and $(2 \pi, 0)$. Notice that the horizontal lifts of $\beta$ are not closed because of the holonomy. 
The Weingarten map, $A$, of $M_{\beta}$ in $S^{3}(1)$ is given by

$$
\begin{aligned}
& A\left(X_{t}\right)=\bar{\lambda} X_{t}+X_{z}, \\
& A\left(X_{z}\right)=X_{t} .
\end{aligned}
$$

Theorem 4. Let $\beta$ be an immersed curve in $S^{2}\left(\frac{1}{2}\right)$ and $M_{\beta}$ its Hopf cylinder in $S^{3}(1)$. Then every geodesic of $M_{\beta}$ is a general helix, lying fully in $S^{3}(1)$.

Proof. Let $\alpha(s)$ be a geodesic of $M_{\beta}$. Then there exists a constant $\varphi$ (the slope) such that

$$
T(s)=\alpha^{\prime}(s)=\cos \varphi X_{z}+\sin \varphi X_{t} .
$$

This equation proves that $T$ makes a constant angle, $\varphi$, with $X_{z}$ which is a Killing vector field of constant magnitude along $\alpha$, and this completes the proof.

We also have a converse of the last theorem,

Theorem 5. Each general helix lying fully in $S^{3}(1)$ can be regarded as a geodesic in a certain Hopf cylinder of $S^{3}(1)$

Proof. Given a general helix, $\gamma$, with curvature, $\kappa$, and torsion, $\tau$, satisfying $\tau=$ $b \kappa \pm 1$ for some constant $b=\cot \theta, \theta$ being the angle between the axis and $\gamma^{\prime}$, we define $\beta$ (up to motions) in $S^{2}\left(\frac{1}{2}\right)$ from its curvature function to be $\lambda=\left(1+b^{2}\right) \kappa \pm 2 b$, and then in $M_{\beta}$ we choose the geodesic $\alpha$ associated with the slope $\varphi$ defined as $\cot \varphi=\mp b$. It is easy to see that $\gamma$ and $\alpha$ have the same curvatures, and so they are congruent in $S^{3}(1)$.

Finally we have the following closedness result.

Corollary 1. Let $\beta$ be an immersed closed curve in $S^{2}\left(\frac{1}{2}\right)$. There exists a rational one-parameter family of closed general helices in $S^{3}(1)$, lying in the Hopf torus $M_{\beta}$

Proof. If $L$ denotes the length of $\beta$ and $A$ the oriented area it encloses in $S^{2}\left(\frac{1}{2}\right)$, then $M_{\beta}$ is isometric to the flat torus obtained from the lattice

$$
\Lambda=\operatorname{Span}\{(2 A, L),(2 \pi, 0)\} .
$$

According to our results, general helices lying in $M_{\beta}$ correspond with geodesics of $M_{\beta}$. Let $\gamma$ be a geodesic of $M_{\beta}$ and, as before, denote its slope by $\varphi$. Then $\gamma$ is closed if and only if there exists $s_{o}>0$ such that $\gamma\left(s_{o}\right)=\gamma(0)$, but the latter holds if and only if $\left(s_{o} \cos \varphi, s_{o} \sin \varphi\right) \in \Lambda$. Consequently, there exists a rational number, $q$, such that

$$
\cot \varphi=\frac{2 A}{L}+q \frac{2 \pi}{L}
$$

\section{REFERENCES}

[BFLM] M.Barros, A.Ferrández, P.Lucas and M.A.Meroño, Helicoidal filaments in the 3-sphere. Preprint.

[Ef] N.V.Efimov, Nekotorye zadachi iz teorii prostranstvennykh krivykh. Usp.Mat.Nauk, 2 (1947), 193-194.

[Fe] W.Fenchel, The differential geometry of closed space curves. Bull.Amer.Math.Soc., 57 (1951), 44-54. MR 12:634d

[L] M.A.Lancret, Mémoire sur les courbes à double courbure. Mémoires présentés à l'Institut 1 (1806), 416-454.

[LP] J.Langer and R.Perline, Local geometric invariants of integrable evolution equations. J.Math.Phys., 35 (1994), 1732-1737. MR 95c:58095 
[LS1] J.Langer and D.A.Singer, The total squared curvature of closed curves. J.Diff.Geom., 20 (1984),1-22. MR 86i:58030

[LS2] J.Langer and D.A.Singer, Knotted elastic curves in $R^{3}$. J.London Math.Soc., 30 (1984), 512-520. MR 87d:53004

[MP] R.S.Millman and G.D.Parker, Elements of Differential Geometry. Prentice-Hall, 1977. MR 56:1208

[O] B.O'Neill, Semi-Riemannian geometry. Academic Press,1983. MR 85f:53002

[Pi] U.Pinkall, Hopf tori in $S^{3}$. Invent.Math., 81 (1985), 379-386. MR 86k:53075

[S] P.D.Scofield, Curves of constant precession. Amer.Math. Monthly, 102 (1995), 531-537. MR 96d:53002

[St] D.J.Struik, Lectures on Classical Differential Geometry. Dover, New-York, 1988. MR 89b:53002

Departamento de Geometria y Topologia, Facultad de Ciencias, Universidade de Granada, 18071 Granada, Spain

E-mail address: mbarros@goliat.ugr.es 